
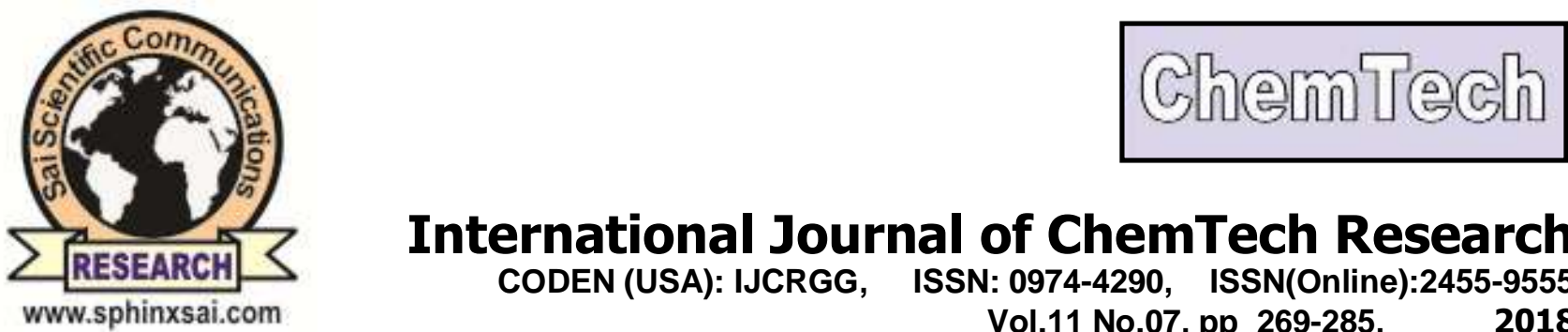

International Journal of ChemTech Research CODEN (USA): IJCRGG, ISSN: 0974-4290, ISSN(Online):2455-9555

Vol.11 No.07, pp 269-285,

2018

\title{
A Review of Advances in Bioethanol Production Processes from Oil Palm Empty Fruit Bunch
}

\author{
Solano $\mathbf{R}^{1 *}$, Rodríguez $\mathbf{M}^{2}$, Ballesteros $\mathrm{K}^{3}$, Jiménez $\mathrm{A}^{4}$ \\ ${ }^{1}$ Multifunctional Nanomaterials Research Group.School of Engineering. Universidad \\ de Cartagena. Street 30 \# 48-152. Cartagena, Colombia. \\ ${ }^{2}$ Design of Processes and Use of Biomass Research Group.School of \\ Engineering.Universidad de Cartagena. Street 30 \# 48-152. Cartagena, Colombia. \\ ${ }^{3}$ Particles and Processes Modeling Research Group.School of \\ Engineering. Universidad de Cartagena. Street 30 \# 48-152. Cartagena, Colombia. \\ ${ }^{4}$ Research Laboratory on Catalysis and New Materials.School of Exact and Natural \\ Sciences.Universidad de Cartagena. Avenue 50 \# 29-11. Cartagena, Colombia.
}

\begin{abstract}
Many studies are carried out in different countries to find alternatives of hydrocarbon-based fuels, 1 which include hydro, wind, biofuels, solar, and geothermal energy. One of the major biofuels isbioethanol, a clear, colorless liquid, biodegradable, and lowin toxicity, and can be considered as a high-octane fuel oroctane enhancer in domestic petrols. The pre-treatment process for oil palm empty fruit bunches (OPEFB) or empty fruit bunches (EFB)for produce bioethanol require convert the complex lignocellulose structures into enzymatically digestible forms and easy handling for use. The pre-treatment can to be divided into physical, chemical, biological and physico-chemical tre-treatment. Various studies on the subject are presented below either a single treatment or combination of several. Nevertheless, the most important problem with bioethanol downstream processing is the dewatering step due to azeotropic formation during distillation of ethanol-water mixtures. Currently, different methods like direct contact membrane distillation (MDBR), extractive batch distillation, pervaporation, adsorption, molecular sieve and pressure Swing Adsorption (PSA) are used for bioethanol dehydration with regard to the absolute ethanol production. Also, mass and energy integration processes are also reviewed.
\end{abstract}

Keywords : bioethanol, pretreatment, process integration, purification.

1. Pretreatment of biomass oil palm empty fruit bunches (OPEFB)

In 2014, Aisyah, Uemuraa and Yusup in Perak, developed a pre-treatment physicochemical where the OPEFB was dried, grinded and passed through a mesh. Later the OPEFB was heated to $180{ }^{\circ} \mathrm{C}$ and then was cooled to $40{ }^{\circ} \mathrm{C}$ and after they added $1.2 \mathrm{~g}$ of $\mathrm{CaO}$ and $\mathrm{NaOH}$ into the EFB and water mixture. Obtaining thus the solid yield of the reaction increased significantly for hydrothermal pretreatment with $\mathrm{NaOH}$ addition (90.08

Solano R et al /International Journal of ChemTech Research, 2018,11(07): 269-285

DOI= http://dx.doi.org/10.20902/IJCTR.2018.110732 
$\%)$ when compared to the hydrothermal pretreatment of EFB (75.51\%) and with addition of $\mathrm{CaO}(73.91 \%)$ [1]. In 2013, in Republic of Korea Kim and Kim soaked the OPEFB in $\mathrm{H}_{2} \mathrm{SO}_{4}$ solution, after in water and dried to $105^{\circ} \mathrm{C}$. Then fiber was soaked in $\mathrm{NaOH}$ solution and dried at $121 \mathrm{C}$ and 5 psi, then to be soaked in water for remove $\mathrm{NaOH}$ on the surface of the biomass repeated times and was dried. The delignification yield after sequential acid/alkali treatment of EPFB fiber was approximately 70\%[2]. Table 1 consolidates conditioning methods and pretreatment of biomass.

Table 1.Methods and pretreatment of biomass

\begin{tabular}{|c|c|c|c|}
\hline Method & Conditions & $\begin{array}{l}\text { Performance lignin } \\
\text { removed }(\%)\end{array}$ & Reference \\
\hline $\begin{array}{l}\text { Pre-treatment } \\
\text { physicochemical } \\
\text { With } \mathrm{NaOH}\end{array}$ & $\begin{array}{l}\text { Drying, grinding and shaking after } \\
\text { alkaline hydrotreatment First heated to } \\
180{ }^{\circ} \mathrm{C} \text {. Cooled } 40{ }^{\circ} \mathrm{C} \text {. Mass of biomass } \\
30 \mathrm{~g} \text {. Volume of water } 300 \mathrm{~mL} \text {. } \\
\text { Reaction time } 10 \text { min. } 1.2 \mathrm{~g} \text { of } \mathrm{NaOH} \text {. }\end{array}$ & $\begin{array}{l}\mathrm{NaOH} \text { addition } \\
(90.08 \%)\end{array}$ & {$[1]$} \\
\hline $\begin{array}{l}\text { Sequential acid/alkali } \\
\text { treatment with } \mathrm{H}_{2} \mathrm{SO}_{4} \\
\text { and } \mathrm{NaOH}\end{array}$ & $\begin{array}{l}\text { Heated at } 105-121^{\circ} \mathrm{C} \text { and } 15 \text { psi for } 24 \\
\text { hrs. } 4 \%(v / v) \mathrm{H}_{2} \mathrm{SO}_{4} \text { solution and } 10 \mathrm{~N} \\
\mathrm{NaOH} \text { solutions. }\end{array}$ & $\begin{array}{l}\text { The delignification } \\
\text { yield was } 70 \% \text {. }\end{array}$ & {$[2]$} \\
\hline $\begin{array}{l}\text { Phosphoric acid } \\
\text { pretreatment and } \\
\text { combined with fungi. }\end{array}$ & $\begin{array}{l}\text { fungus Pleurotusfloridanus to } 31^{\circ} \mathrm{C} \text { and } \\
\text { neutral PH, } 8 \mathrm{ml} \text { phosphoric acid } \\
(85.7 \%) \text {, Washed with } 40 \mathrm{~mL} \text { acetone } \\
\text { and centrifuged at } 1900 \mathrm{~g}\end{array}$ & $\begin{array}{l}\text { Phosphoric acid } \\
\text { pretreatment } 89.4 \% \\
\text { and combined with } \\
\text { lignin yield of } \\
62.8 \% \text {. } \\
\end{array}$ & {$[3]$} \\
\hline $\begin{array}{l}\text { Chemical pretreated } \\
\text { with aqueous ammonia }\end{array}$ & $\begin{array}{l}60 \mathrm{C}, 12 \mathrm{~h}, \text { and } 21 \%(\mathrm{w} / \mathrm{w}) \text { aqueous } \\
\text { ammonia }\end{array}$ & $\begin{array}{l}41.1 \% \text { lignin } \\
\text { removal }\end{array}$ & [4] \\
\hline $\begin{array}{l}\text { Acid pretreatment with } \\
\text { sulfuric acid }\end{array}$ & $\begin{array}{l}1 \%(\mathrm{w} / \mathrm{v}) \text { sulfuric acid, to } 190 \mathrm{C} \text { and } \\
\text { dried at } 45 \mathrm{C} \text { for more than } 3 \text { days }\end{array}$ & $\begin{array}{l}\text { decreasing } 90 \% \text { the } \\
\text { lignite content in } \\
\text { sample }\end{array}$ & {$[5]$} \\
\hline $\begin{array}{l}\text { Alkaline } \\
\text { hydrotreatment with } \\
\mathrm{NaOH}\end{array}$ & $\begin{array}{l}95 \mathrm{~g} \mathrm{NaOH}(8 \% \mathrm{p} / \mathrm{v}) \text {, for heated at } 100 \\
\mathrm{C} \text { for } 10,20 \text { and } 60 \mathrm{~min} \text {, dried at } \\
10000 \mathrm{rpm}\end{array}$ & $\begin{array}{l}\text { conversions of } \\
\text { lignin solids of } 96 \%\end{array}$ & {$[6]$} \\
\hline $\begin{array}{l}\text { Physical and } \\
\text { hydrotreatment } \\
\text { simultaneously with } \\
\mathrm{NaOH} \text { and } \mathrm{CaOH} \text { with } \\
\mathrm{H}_{2} \mathrm{O}_{2}\end{array}$ & $\begin{array}{l}\text { Dry at } 105 \mathrm{C} \text { for } 24 \text { hrs. } \\
\text { to } 5 \text { gr sample, concentrations } 100 \\
\text { mMNaOH and } \mathrm{CaOH} \text { with } \mathrm{H}_{2} \mathrm{O}_{2} \\
\text { and dried } 100{ }^{\circ} \mathrm{C}\end{array}$ & $\begin{array}{l}\text { Almost } 100 \% \text { of } \\
\text { lignin. }\end{array}$ & [7] \\
\hline $\begin{array}{l}\text { Phisycal and Chemical } \\
\text { treatment with NAOH } \\
\text { and irradiation }\end{array}$ & $\begin{array}{l}\text { Dried and milled } \\
\mathrm{NaOH} 10 \%, 150{ }^{\circ} \mathrm{C} \text {, ratio 5:1 } \mathrm{NAOH} \\
\text { and } \mathrm{EFB} \text { and irradiation } 8 \text { (energy } \\
\text { variation of } 100 \mathrm{kGy} \text { up to } 500 \mathrm{kGy} \text { ) }\end{array}$ & $\begin{array}{l}92,2 \% \text { total lignin } \\
\text { removed }\end{array}$ & {$[8]$} \\
\hline $\begin{array}{l}\text { Pretreatment dilute } \\
\text { acid }\left(\mathrm{H}_{2} \mathrm{SO}_{4}\right)\end{array}$ & $\begin{array}{l}\text { Optimal condition } 161.5 \mathrm{C}, 9.44 \mathrm{~min} \\
\text { and } 1.51 \% \text { acid loading }\end{array}$ & $\begin{array}{l}\text { Content of lignin } \\
\text { removed of sample } \\
\text { to } 43 \% \text { lignin yield }\end{array}$ & [9] \\
\hline $\begin{array}{l}\text { Steam explosion (SE) } \\
\text { pretreatment }\end{array}$ & $\begin{array}{l}300 \text { gr of OPEFB was dried at } 65^{\circ} \mathrm{C} \text { for } \\
72 \mathrm{~h} \text {. saturated with steam to } 195^{\circ} \mathrm{C} \text { for } \\
6 \mathrm{~min} .\end{array}$ & $\begin{array}{l}\text { Lignin analyses } \\
\text { showed a reduction } \\
\text { of } 68.12 \%\end{array}$ & {$[10]$} \\
\hline $\begin{array}{l}\text { Physical and alkaline } \\
\text { treatment combined } \\
\text { with } \mathrm{NaOH}\end{array}$ & $\begin{array}{l}\text { Dried at } 65{ }^{\circ} \mathrm{C} \text { for } 48 \mathrm{~h} \text {, milled, sieved } \\
\text { through a mesh } 42(0.350 \mathrm{~mm}) \\
\mathrm{NaOH} \text { of } 0,5 \text { to } 5,5 \% \text { in solution at } 121 \\
{ }^{\circ} \mathrm{C} \text { and } 80 \mathrm{~min}\end{array}$ & $\begin{array}{l}\text { Presence of lignin } \\
\text { decayed in a } 70 \% .\end{array}$ & {$[11]$} \\
\hline $\begin{array}{l}\text { Chemical pre- } \\
\text { treatment with } \mathrm{NaOH} \\
\text { and mechanical pre- } \\
\text { treatment }\end{array}$ & $\begin{array}{l}3 \% \mathrm{NaOH}, 110 \mathrm{C} \text { for } 45 \mathrm{~min} \text {. milled to } \\
\text { average } 1 \mathrm{~mm} \text { and washed with water }\end{array}$ & $85 \%$ lignin removal & [12] \\
\hline
\end{tabular}




\begin{tabular}{|c|c|c|c|}
\hline $\begin{array}{l}\text { Physical and chemical } \\
\text { pretreatment }\end{array}$ & $\begin{array}{l}\text { Washed, defibrated and ground. AFEX } \\
\text { at } 135^{\circ} \mathrm{C}, 45 \mathrm{~min} \text { retention time. }\end{array}$ & $\begin{array}{l}\text { Particle size was } \\
\text { reduced }\end{array}$ & [13] \\
\hline $\begin{array}{l}\text { Chemical pretreatment } \\
\text { with } \mathrm{NaOH}\end{array}$ & $\begin{array}{l}\mathrm{NaOH} 127.64{ }^{\circ} \mathrm{C}, 22.08 \mathrm{~min}, \text { and } 2.89 \\
\mathrm{~mol} \mathrm{~L}^{-1}\end{array}$ & $\begin{array}{l}74,33 \% \text { lignin } \\
\text { removal }\end{array}$ & [14] \\
\hline $\begin{array}{l}\text { Physical and acid } \\
\text { pretreatment }\end{array}$ & $\begin{array}{l}\text { Dried and milled. The sulfuric acid at } \\
100{ }^{\circ} \mathrm{C} \text { to } 150{ }^{\circ} \mathrm{C} \text {, time ranged from } 30 \\
\text { to } 90 \mathrm{~min} \text {, and acid loading } 0 \text { to } 1.3 \% \\
\text { weight acid/weight liquid. }\end{array}$ & $\begin{array}{l}63 \% \text { total lignin } \\
\text { removed }\end{array}$ & [15] \\
\hline $\begin{array}{l}\text { Chemical pretreatment } \\
\text { with Ethanol/benzene, } \\
\mathrm{NaClO}_{2}, \mathrm{KOH} \text { and } \\
\text { deionized water }\end{array}$ & $\begin{array}{l}\text { The EFB of } 0,5-1 \mathrm{~cm} \text {. Ethanol/benzene } \\
(1: 2 \mathrm{v} / \mathrm{v}) \mathrm{mixed} \text { solvent. } \mathrm{NaClO}_{2} \\
\text { solution at }(\mathrm{pH} 4-5) \text { at } 70 \mathrm{C} \text { for } 1 \mathrm{~h} .6 \\
\mathrm{wt} \% \mathrm{KOH} \text { solution at } 20^{\circ} \mathrm{C} \text { for } 24 \mathrm{~h} \text {. } \\
\text { deionized water until the } \mathrm{pH} 7\end{array}$ & $\begin{array}{l}\text { Average thickness } \\
\text { of nanofibers was } \\
\text { within the range 1- } \\
3.5 \mathrm{~nm}\end{array}$ & [16] \\
\hline $\begin{array}{l}\text { Biological } \\
\text { pretreatment }\end{array}$ & $\begin{array}{l}\text { Six days at } 30^{\circ} \mathrm{C} \text { cultivated. } P . \\
\text { ostreatus CECT } 20311 \text { fungi }\end{array}$ & $\begin{array}{l}\text { The lignin } \\
\text { degradation to } 50 \% \\
\text { with } P \text {. ostreatus, a } \\
\text { higher value than the } \\
41 \% \text { reached with } P \text {. } \\
\text { chrysosporium }\end{array}$ & [17] \\
\hline $\begin{array}{l}\text { Chemical pretreatment } \\
\text { (Ozone treated) with } \\
\mathrm{NaOH}\end{array}$ & $\begin{array}{l}\text { For ozonetreated: } 100 \mathrm{~mL} \text { of } \mathrm{NaOH}(5 \\
\text { wt. } \% \text { ) for } 1 \mathrm{~h} \text {. washed with distilled } \\
\text { water. dried in the oven at } 105{ }^{\circ} \mathrm{C} \text { for } \\
50 \mathrm{~min}\end{array}$ & $\begin{array}{l}\text { lignin degradation of } \\
84.7 \mathrm{wt} . \%\end{array}$ & [18] \\
\hline Bisulfite pretreatment & $\begin{array}{l}\text { Pretreated samples were washed and } \\
\text { Five oxygen-catalyzed at } 0.6 \mathrm{MPa} \text { and } \\
30 \text { min at } 120^{\circ} \mathrm{C}\end{array}$ & $\begin{array}{l}\text { Lignin removed } \\
75 \%\end{array}$ & [19] \\
\hline $\begin{array}{l}\text { Phisycal and Bisulfite } \\
\text { pretreatment }\end{array}$ & $\begin{array}{l}\text { Milled to particle sizes ranging from } \\
0.30 \text { to } 0.45 \mathrm{~mm} \text {. Pretreated samples } \\
\text { were washed and Five oxygen- } \\
\text { catalyzed at } 0.6 \mathrm{MPa} \text { and } 30 \mathrm{~min} \text { at } 120 \\
{ }^{\circ} \mathrm{C}\end{array}$ & $\begin{array}{l}\text { Lignin removed } \\
79,6 \%\end{array}$ & [20] \\
\hline Bisulfite pretreatment & $\begin{array}{l}\text { The bisulfite pretreatment at }(180 \mathrm{C}, 30 \\
\left.\text { min, } 8 \% \mathrm{NaHSO}_{3}, 1 \% \mathrm{H}_{2} \mathrm{SO}_{4}\right) \text {. Reacted } \\
\text { with a solution of sodium bisulfite at } \\
180 \mathrm{C} \text { for } 30 \text { min, at } 8 \% \text { and } 10 \% \\
\mathrm{NaHSO}_{3}\end{array}$ & $\begin{array}{l}\text { Lignin removed } \\
79,1 \%\end{array}$ & [21] \\
\hline $\begin{array}{l}\text { Phisycal pretreatment } \\
(\text { Ball milling }(\mathrm{BM}))\end{array}$ & 6-24 h, constant speed of $230 \mathrm{rpm}$ & $\begin{array}{l}\text { Lignin removed } \\
81,32 \%\end{array}$ & {$[22]$} \\
\hline $\begin{array}{l}\text { Phisycal, chemical and } \\
\text { hydrothermal } \\
\text { treatment, combined. }\end{array}$ & $\begin{array}{l}\text { Crushed particle size } 5 \mathrm{~mm} .1 \% \mathrm{NaOH} \\
(\mathrm{w} / \mathrm{w}) \text {. team treated at } 230 \mathrm{C} \text { for } 15 \mathrm{~min} \\
\text { in pressure vessel }\end{array}$ & $\begin{array}{l}\text { Lignin decrease } \\
\text { until } 80 \%\end{array}$ & [23] \\
\hline $\begin{array}{l}\text { High-pressure steam } \\
\text { pre-treatment (HPST) }\end{array}$ & $\begin{array}{l}\text { Press-shredded at } 250{ }^{\circ} \mathrm{C} \text { and } 9.4 \mathrm{MPa} \text {. } \\
\text { HPST conditions of } 170 / 0.82,190 / 1.32 \text {, } \\
210 / 2.03 \text {, and } 230{ }^{\circ} \mathrm{C} / 3.00 \mathrm{MPa} \text { for } 2 \text {, } \\
4,8 \text {, and } 10 \mathrm{~min} \text {. oven-dried at } 105^{\circ} \mathrm{C} \\
\text { for } 24 \mathrm{~h}\end{array}$ & $\begin{array}{l}\text { Lignin reduction of } \\
83 \% \text {. }\end{array}$ & [24] \\
\hline
\end{tabular}




\begin{tabular}{|c|c|c|c|}
\hline $\begin{array}{l}\text { Chemical pretreatment } \\
\text { (organosolv } \\
\text { pretreatment) }\end{array}$ & $\begin{array}{l}\text { Aqueous ethanol } 1: 10(10 \mathrm{~g} \text { in } 100 \\
\mathrm{mL}) \text {. Concentration }(35,55, \text { and } 75 \% \\
\text { vol), at reaction temperature }(80,100, \\
\left.\text { and } 120{ }^{\circ} \mathrm{C}\right) \text { and reaction time }(30,60 \text {, } \\
\text { and } 90 \mathrm{~min}) .\left(\mathrm{KMnO}_{4}\right) 0.1 \mathrm{~N} \text {, Sulfuric } \\
\text { acid }\left(\mathrm{H}_{2} \mathrm{SO}_{4}\right) 4.0 \mathrm{~N} \text { and Potassium } \\
\text { iodide }(\mathrm{KI}) \text { for } 10 \text { minutes }\end{array}$ & $\begin{array}{l}\text { Decrease lignin } \\
\text { concentration of } \\
75 \%\end{array}$ & {$[25]$} \\
\hline $\begin{array}{l}\text { Alkaline pretreatment } \\
\text { with } \mathrm{NaOH} \text { and steam. }\end{array}$ & $\begin{array}{l}\text { Wash EFB with } \mathrm{NaOH} 2 \%, 4 \mathrm{~h} \text { at } 30 \\
{ }^{\circ} \mathrm{C} \text {, with solid to liquid ratio of } 1: 10 . \\
\text { Heating at } 121 \mathrm{C} \text { and } 117 \mathrm{kPa} \text { during } 6 \\
\text { min }\end{array}$ & $\begin{array}{l}\text { Lignin removed } 92.3 \\
\% \text {. }\end{array}$ & {$[26]$} \\
\hline $\begin{array}{l}\text { Chemical pretreatment } \\
\text { with sulfuric acid }\end{array}$ & $\begin{array}{l}\text { Air-dried and pretreated at } 170 \mathrm{C} \text { with } \\
0.8 \mathrm{wt} \% \text { sulfuric acid and a solid/liquid } \\
\text { ratio of } 1: 6 . \text { stirring speed } 100 \mathrm{rpm} \text { and } \\
15 \mathrm{~min}\end{array}$ & $\begin{array}{l}\text { lignin content } \\
\text { decreased }\end{array}$ & {$[27]$} \\
\hline $\begin{array}{l}\text { Ultrasonic pre- } \\
\text { treatment with } \mathrm{H}_{2} \mathrm{SO}_{4}\end{array}$ & $\begin{array}{l}500 \mathrm{ml} \text { of } 2 \% \mathrm{H}_{2} \mathrm{SO}_{4} \text { with } 50 \mathrm{~g} \text { of } \\
\text { OPEFB. Ultrasonicated at a power of } 2 \\
\mathrm{~kW}, 20 \mathrm{kHz} \text { for } 15,60 \text { and } 45 \mathrm{~min} \text {, and } \\
\text { amplitude of study was } 15 \%, 60 \% \text { and } \\
90 \%\end{array}$ & $\begin{array}{l}\text { Lignin removed } 81,9 \\
\% \text {. }\end{array}$ & {$[28]$} \\
\hline $\begin{array}{l}\text { Alkaline pre- } \\
\text { treatments }\end{array}$ & $\begin{array}{l}\text { Washed, air-dried and refined to size of } \\
\text { about } 2-4 \mathrm{~cm} \text {. applied pre-treatments at } \\
\text { liquid/solid ratio } 12: 1 \text { for } 60 \mathrm{~min} \text {, } \\
\text { Sodium Hydroxide }(\mathrm{NaOH}) 2 \% \mathrm{w} / \mathrm{v} \text {, } \\
120^{\circ} \mathrm{C} \text {. The fibers were washed and } \\
\text { spin-dried }\end{array}$ & $\begin{array}{l}\text { Lignin removed 91,3 } \\
\% .\end{array}$ & [29] \\
\hline $\begin{array}{l}\text { Sequential } \\
\text { pretreatment (Phisycal, } \\
\text { dilute acid and alkali } \\
\text { pretreatment) }\end{array}$ & $\begin{array}{l}\text { Washed and dried at } 90 \mathrm{C} \text { for } 24 \mathrm{~h} \text {. } \\
\text { Dilute sulfuric acid at concentration of } \\
0,1-8,0 \%(\mathrm{v} / \mathrm{v}) \text { at } 121 \mathrm{C}, 15 \text { psi for } 1 \mathrm{~h} \text {, } \\
10 \mathrm{~N} \mathrm{NaOH} \text { solution at ambient } \\
\text { temperature for } 4 \mathrm{~h} \text {, then, was heated at } \\
121 \mathrm{C}, 15 \text { psi for } 15 \mathrm{~min}\end{array}$ & $\begin{array}{l}\text { Removed } 70 \% \\
\text { lignin. }\end{array}$ & {$[30]$} \\
\hline
\end{tabular}

\section{Purification process in bioethanol production}

Jeon et al. [31] evaluated the production of anhydrous ethanol using oil palm empty fruit bunch in a pilot plant by distillation and dehydration process. The initial conditions were $92-93{ }^{\circ} \mathrm{C}$ and $16-20 \%$ wt. After the ethanol was refluxed to the top portion of the rectification column. Operation conditions were a $1 / 10$ reflux ratio, $77-78{ }^{\circ} \mathrm{C}$ top portion temperature, and $100.5{ }^{\circ} \mathrm{C}$ bottom portion temperature. Consequently, $93.7 \mathrm{wt} \%$ ethanol was produced by the authors.Subsequently, the pressure swing adsorption (PSA, dehydration) process was applied in this study.The dehydration process was operated at a feed/purge ratio of $7 / 3$ for the stable production of anhydrous ethanol. Consequently, $99.6 \%$ wt ethanol was produced. Coelho et al. [32] studied the bioethanol dehydration using natural clinoptilolite. The results showed that by working at optimum condition, feed with $96 \%(\mathrm{v} / \mathrm{v})$ initial ethanol concentration could be purified up to $99.9 \%(\mathrm{v} / \mathrm{v})$. In addition, the optimum operational conditions to reach $99.9 \%$ for final ethanol concentration were found equal to $10.7{ }^{\circ} \mathrm{C}, 4.9$ bar and 8 $\mathrm{mL} / \mathrm{min}$ for liquid temperature, pressure and flow rate, respectively. Table 2 shows the advances for the purification of bioethanol. 
Table 2. Advances for bioethanol purification: Review.

\begin{tabular}{|c|c|c|c|}
\hline Method & Initial experimental conditions & $\begin{array}{l}\text { Result of purification } \\
\text { process }\end{array}$ & Reference \\
\hline $\begin{array}{l}\text { Distillation and } \\
\text { dehydration } \\
\text { (pressure swing } \\
\text { adsorption). }\end{array}$ & $\begin{array}{l}\text { Distillation: } 92-93{ }^{\circ} \mathrm{C} \text { and } 16-20 \\
\% \text { wt. } \\
\text { Dehydration: feed/purge ratio of } \\
7 / 3 \text {. }\end{array}$ & $99.6 \% \mathrm{wt}$ & Jeon et al. [31]. \\
\hline $\begin{array}{l}\text { Dehydration } \\
\text { (natural } \\
\text { clinoptilolite). }\end{array}$ & $\begin{array}{l}96 \%(\mathrm{v} / \mathrm{v}) 10.7{ }^{\circ} \mathrm{C}, 4.9 \text { bar and } 8 \\
\mathrm{~mL} / \mathrm{min} .\end{array}$ & $99.9 \%(\mathrm{v} / \mathrm{v})$ & Coelho et al. [32]. \\
\hline $\begin{array}{l}\text { Direct contact } \\
\text { membrane } \\
\text { distillation } \\
\text { (MDBR). }\end{array}$ & $\begin{array}{l}24 \mathrm{~h} \text { of the fermentation using } \\
\text { whey enriched with sucrose (100 } \\
\left.\mathrm{g} \mathrm{dm}^{-3}\right) \text {. }\end{array}$ & $53.74 \mathrm{~g} \mathrm{dm}^{-3}$ & $\begin{array}{l}\text { Tomaszewska and } \\
\text { Białończyk [33]. }\end{array}$ \\
\hline $\begin{array}{l}\text { Direct Contact } \\
\text { Membrane } \\
\text { Distillation } \\
\text { (DCMD). }\end{array}$ & $\begin{array}{l}490 \mathrm{~cm}^{2} \text {, effective membrane. for } \\
860 \mathrm{~h} \text {. }\end{array}$ & $\begin{array}{l}\text { Ethanol flux } 1-4 \quad(\mathrm{~kg} \\
\text { EtOH }) / \mathrm{m}^{2} \text { per day }\end{array}$ & $\begin{array}{l}\text { Barancewicz and } \\
\text { Gryta [34]. }\end{array}$ \\
\hline $\begin{array}{l}\text { Direct contact } \\
\text { membrane } \\
\text { distillation } \\
\text { (DCMD). }\end{array}$ & $\begin{array}{l}\mathrm{PP}, 0.2 \mu \mathrm{m} . \Delta \mathrm{T} \text { between } 13 \text { and } \\
20{ }^{\circ} \mathrm{C} .3 \mathrm{~h} \text { using clean } 10 \mathrm{wt} . \% \\
\text { ethanol feed. }\end{array}$ & $\begin{array}{l}\text { Ethanol flux in permeate of } \\
1.2 \mathrm{~L} \mathrm{~m}^{-2} \mathrm{~h}^{-1}\end{array}$ & $\begin{array}{l}\text { Lewandowicz et } \\
\text { al. [35]. }\end{array}$ \\
\hline $\begin{array}{l}\text { Membrane } \\
\text { (capillary) } \\
\text { distillation. }\end{array}$ & $\begin{array}{l}\text { Porosity of } 70 \% \text { and the effective } \\
\text { area of } 0.0183 \mathrm{~m}^{2} . \quad \text { Feed } \\
\text { temperature of } 310 \mathrm{~K} .\end{array}$ & $2.5-4 \mathrm{~g} \mathrm{dm}^{-3} \mathrm{~h}^{-1}$ & $\begin{array}{l}\text { Tomaszewska and } \\
\text { Białończyk [36]. }\end{array}$ \\
\hline $\begin{array}{l}\text { Membrane } \\
\text { distillation (MD- } \\
\text { DHN-BP } \\
\text { system). }\end{array}$ & $\begin{array}{l}\text { Ethanol feed (65\% wt.) and } 45 \\
{ }^{\circ} \mathrm{C}\end{array}$ & $87 \%$ wt. & $\begin{array}{l}\text { Woldemariam et } \\
\text { al. [37]. }\end{array}$ \\
\hline $\begin{array}{l}\text { Membrane } \\
\text { distillation } \\
\text { (SGMD). }\end{array}$ & $\begin{array}{l}\text { PTFE, } 169 \mathrm{~cm}^{2} \text { active area, } 0.22 \\
\mu \mathrm{m} \text { pore size and porosity of } \\
\left.70 \% \text {. flow rate }(600 \mathrm{~mL} \mathrm{~min})^{-1}\right) \text {, } \\
\text { feed concentrations }(7 \% \mathrm{wt}) \text {, flow } \\
\text { rate of the sweeping air }(4.63 \mathrm{NL} \\
\left.\text { min }^{-1}\right) \text {, temperature of the feed } \\
\text { solution }\left(55^{\circ} \mathrm{C}\right) \text { and temperature } \\
\text { of the sweeping air }\left(25 \pm 1^{\circ} \mathrm{C}\right) \text {. }\end{array}$ & $\begin{array}{l}\text { Ethanol flux of } 1.8 \mathrm{~L} \mathrm{~m}^{-2} \mathrm{~h}^{-1} \\
\text { in the permeated. }\end{array}$ & Mahdi et al. [38]. \\
\hline $\begin{array}{l}\text { Vacuum } \\
\text { membrane } \\
\text { distillation (VHG } \\
+ \text { VMD). }\end{array}$ & $300 \mathrm{~g} / \mathrm{L}$ glucose loading. & $\begin{array}{l}127.4 \mathrm{~g} / \mathrm{L}(16.1 \% \mathrm{v} / \mathrm{v}) \\
\text { ethanol and permeate flux } \\
\text { of } 63.7 \mathrm{~g} / \mathrm{L}-\mathrm{h} .\end{array}$ & Zhang et al. [39]. \\
\hline $\begin{array}{l}\text { Vacuum } \\
\text { membrane } \\
\text { distillation } \\
\text { (VMD). }\end{array}$ & $\begin{array}{l}\text { PVDF membranes }(0.18 \mu \mathrm{m} \text { and } \\
85 \% \text {, porosity). It was observed } \\
\text { that feed velocity and temperature } \\
\text { had a significant effect on VMD. } \\
0.6 \mathrm{~m} / \mathrm{s}-1.1 \mathrm{~m} / \mathrm{s} \text { (feed velocity) } \\
\text { and temperature from } 50{ }^{\circ} \mathrm{C} \text { to } 70 \\
{ }^{\circ} \mathrm{C}\end{array}$ & $\begin{array}{l}4.6 \mathrm{~L} \mathrm{~m}^{-2} \mathrm{~h}^{-1} \text { to } 9.5 \mathrm{~L} \mathrm{~m}^{-2} \mathrm{~h}^{-} \\
1 \text {, distillation flux. }\end{array}$ & Zhang et al. [40]. \\
\hline $\begin{array}{l}\text { Extractive batch } \\
\text { distillation (semi- } \\
\text { continuous). }\end{array}$ & $\begin{array}{l}\text { Dividing wall distillation column } \\
\text { using glycerol as entrainer. } \\
\text { ethanol-water mixture of } \\
\text { composition } 92 \% \text { wt.The glycerol } \\
\text { was supplied at } 60^{\circ} \mathrm{C} \text {. }\end{array}$ & $99 \% \mathrm{wt}$. & García et al. [41]. \\
\hline
\end{tabular}




\begin{tabular}{|c|c|c|c|}
\hline $\begin{array}{l}\text { Extractive batch } \\
\text { distillation. }\end{array}$ & $\begin{array}{l}\text { Glycerol charge }(2.0: 1.0) \\
\text { Mixture of ethanol-water }(94 \% \\
\text { wt.) and ambient pressure }(604 \\
\mathrm{mmHg}) \text {. }\end{array}$ & $98 \% \mathrm{wt}$. & $\begin{array}{l}\text { Navarrete et al. } \\
\text { [42]. }\end{array}$ \\
\hline $\begin{array}{l}\text { Extractive batch } \\
\text { distillation. }\end{array}$ & $\begin{array}{l}\text { Ethanol and water ( } 85 \% \text { in mass } \\
\text { of ethanol). The batch distillation } \\
\text { column operated at atmospheric } \\
\text { pressure }(604 \mathrm{mmHg}) \text {. }\end{array}$ & $\begin{array}{l}0.96 \text { mol fraction of } \\
\text { ethanol. }\end{array}$ & $\begin{array}{l}\text { Pacheco-Basulto et } \\
\text { al. [43]. }\end{array}$ \\
\hline $\begin{array}{l}\text { Pervaporative } \\
\text { dehydration. }\end{array}$ & $\begin{array}{l}\text { PVA membranes (membrane area } \\
\left.\text { of } 2 \mathrm{~m}^{2}\right) \text {.Permeate side pressure } \\
(<10 \mathrm{mbar}) \text {, Product flow } \\
\text { pressure }(2.5 \text { bar }) \text {, Feed flow rate } \\
\left(20 \mathrm{~L} \mathrm{~h}^{-1}\right) \text {, Temperature of the } \\
\text { feed }\left(98{ }^{\circ} \mathrm{C}\right) \text { and Feed ethanol } \\
(85 \% \mathrm{wt} .) .\end{array}$ & $99.7 \%$ wt. & $\begin{array}{l}\text { Niemistö et al. } \\
\text { [44]. }\end{array}$ \\
\hline $\begin{array}{l}\text { Fermentation- } \\
\text { pervaporation. }\end{array}$ & $\begin{array}{l}\text { Polydimethylsiloxane (PDMS) } \\
\text { membrane. The continuous } \\
\text { process evaluated by the authors } \\
\text { had a duration of } 210 \mathrm{~h} .\end{array}$ & $\begin{array}{l}446.3 \mathrm{~g} / \mathrm{L} \text { of ethanol, with } \\
\text { ethanol separation factor } \\
\text { ranging from } 8 \text { to } 12 \text { and } \\
\text { ethanol flux between } 202.2- \\
299.7 \mathrm{~g} / \mathrm{m}^{2} \mathrm{~h} .\end{array}$ & Fu et al. [46]. \\
\hline $\begin{array}{l}\text { Pervaporative } \\
\text { dehydration. }\end{array}$ & $\begin{array}{l}\text { Polydimethylsiloxane/polyetheri } \\
\text { mide (PDMS/PEI) composite. } \\
\text { The permeate pressure maintained } \\
\text { below } 5 \mathrm{~mm} \mathrm{Hg} \text {. Feed } \\
\text { temperature: } 20{ }^{\circ} \mathrm{C} \text { to } 60{ }^{\circ} \mathrm{C} \text { and } \\
\text { ethanol initialconcentrations of } 1- \\
10 \% \text { was studied. }\end{array}$ & $\begin{array}{l}\text { Total flux of } 231-252 \mathrm{~g} / \mathrm{m}^{2} \\
\mathrm{~h} \text {. }\end{array}$ & Lee et al. [47]. \\
\hline Pervaporation. & $\begin{array}{l}\text { vinyltriethoxysilane (VTES)-g- } \\
\text { silicalite-1/PDMS/PAN thin-film } \\
\text { composite membrane }(72.34 \\
\left.\mathrm{cm}^{2}\right) \text {. Temperature of } 35{ }^{\circ} \mathrm{C}, \text { feed } \\
\text { flow rate was kept at } 2.0 \mathrm{~L} / \mathrm{min} \text {, } \\
\text { downstream pressure was } \\
\text { maintained at } 210 \mathrm{~Pa} \text { and the feed } \\
\text { concentrations of } 1.6-8.8 \% \text { wt. } \\
\end{array}$ & $\begin{array}{l}\text { 22.1-61.4 \%wt. } \\
\text { Separation factor of 7-14. }\end{array}$ & Yi and Wan [48]. \\
\hline $\begin{array}{l}\text { Fermentation } \\
\text { coupled with } \\
\text { pervaporation. }\end{array}$ & $\begin{array}{l}\text { Three runs of fermentation- } \\
\text { pervaporation experiment was } \\
\text { carried out lasting for } 192 \mathrm{~h}, 264 \\
\mathrm{~h} \text { and } 360 \mathrm{~h} \text {. Fermentation broth } \\
\text { temperature of } 35^{\circ} \mathrm{C} \text { and feed } \\
\text { concentration between } 10-61 \mathrm{~g} \mathrm{~L}^{-} \\
1 .\end{array}$ & $\begin{array}{l}\text { The total flux of the } \\
\text { polydimethylsiloxane } \\
\text { (PDMS) membrane: } 350 \mathrm{~g} \\
\mathrm{~m}^{2} \mathrm{~h}^{-1}-600 \mathrm{~g} \mathrm{~m}^{2} \mathrm{~h}^{-1} \text {. }\end{array}$ & Fan et al. [49]. \\
\hline Pervaporation. & $\begin{array}{l}\text { PDMS membranes. } 3 \% \text { wt. } \\
\text { ethanol in the feed mixture, The } \\
\text { feed flow rate and temperature } \\
\text { were } 20 \mathrm{~L} \mathrm{~h}^{-1} \text { to } 80 \mathrm{~L} \mathrm{~h}^{-1} \text { and } 22 \\
{ }^{\circ} \mathrm{C} \text {, respectively, with a permeate } \\
\text { pressure of less than } 5 \mathrm{mmHg} \text {. }\end{array}$ & $\begin{array}{l}\text { The mass flux of permeate, } \\
\text { enrichment factor and } \\
\text { permeate concentration } \\
\text { achieved by authors were } \\
5.85 \mathrm{~g} \mathrm{~m}^{-2} \mathrm{~h}^{-1}, 8.8,18.18 \\
\% \mathrm{wt}, \text { respectively. } \\
\end{array}$ & Bello et al. [50]. \\
\hline $\begin{array}{l}\text { Pervaporative } \\
\text { dehydration. }\end{array}$ & $\begin{array}{l}\text { PVA and methylated silicium } \\
\text { membrane. Feed concentration of } \\
94 \% \text { wt, permeate pressure of } 300 \\
\mathrm{~Pa} \text { and } 60^{\circ} \mathrm{C} \text {. }\end{array}$ & 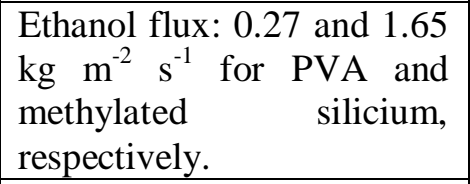 & Moussa et al. [51]. \\
\hline $\begin{array}{l}\text { Pervaporative } \\
\text { dehydration. }\end{array}$ & $\begin{array}{l}\text { Chitosan-Alginate/PES } \\
\text { membranes. } \\
\text { Feed concentration: } 95.5 \% \text { wt. }\end{array}$ & $99.6 \%$ wt. & Asih et al. [52]. \\
\hline
\end{tabular}




\begin{tabular}{|c|c|c|c|}
\hline Pervaporation. & $\begin{array}{lrr}\text { polyamide } & \text { thin-film } & \text { composite } \\
\text { membranes } & \left(10.24 \mathrm{~cm}^{2},\right. & \text { effective } \\
\text { area) with } & \text { modified } \\
\text { polyacrylonitrile. } & \text { Feed } \\
\text { conditions: } 90 \quad \text { wt } \% & \text { aqueous } \\
\text { ethanol solution at } 25^{\circ} \mathrm{C} & \\
\end{array}$ & $99.5 \mathrm{wt} \%$ & Huang et al. [53]. \\
\hline $\begin{array}{l}\text { Vapor phase } \\
\text { dehydration } \\
\text { (dehydration } \\
\text { column). }\end{array}$ & $\begin{array}{l}\text { Dehydration of ethanol was } \\
\text { conducted on dehydration column } \\
\text { with a length of } 30 \mathrm{~cm} \text { and a } \\
\text { diameter of } 2 \text { inches. }\end{array}$ & Above $99 \%$ wt. & Krido et al. [54]. \\
\hline $\begin{array}{l}\text { Pervaporation } \\
\text { dehydration. }\end{array}$ & $\begin{array}{l}\text { Ultrathin zeolite } \mathrm{X} \text { membranes } \\
\left(3.14 \mathrm{~cm}^{2}\right) . \quad 90 / 10 \quad \% \text { wt. } \\
\text { ethanol/water mixture by } \\
\text { pervaporation at } 40-65 \quad{ }^{\circ} \mathrm{C} \text {, } \\
\text { permeate side pressure between } \\
0.7-1.2 \mathrm{kPa} \text { and flow rate of } 0.7 \\
\mathrm{dm}^{3} \mathrm{~min}^{-1} \text {. }\end{array}$ & $\begin{array}{l}\text { Total flux of } 3.37 \pm 0.08 \mathrm{~kg} \\
\mathrm{~m}^{-2} \mathrm{~h}^{-1} \text { and a separation } \\
\text { factor of } 296 \pm 4 .\end{array}$ & Zhou et al. [55]. \\
\hline Adsorption. & $\begin{array}{l}1 \mathrm{ml} \text { of the azeotrope ethanol } \\
\text { water mixture was added to } 0.3 \mathrm{~g} \\
\text { of zeolite } \mathrm{Na}-\mathrm{A} \text { in } 1.5 \mathrm{~mL} \text { vial } \\
\text { with a contact time of } 1 \mathrm{~h} \text {. }\end{array}$ & $\begin{array}{l}\text { The maximum water } \\
\text { adsorption capacity was } \\
100 \mathrm{mg} \text { of water for } 1 \mathrm{~g} \text { of } \\
\text { adsorbent used. }\end{array}$ & $\begin{array}{l}\text { Abdullah et al. } \\
\text { [56]. }\end{array}$ \\
\hline $\begin{array}{l}\text { Purification via } \\
\text { dehydration. }\end{array}$ & $\begin{array}{l}\text { Natural clinoptilolite phase } \\
\text { dehydration using natural zeolite } \\
\text { (clinoptilolite). Feed with } 96 \% \\
(\mathrm{v} / \mathrm{v}) \text { initial ethanol concentration. } \\
10.7{ }^{\circ} \mathrm{C}, 4.9 \text { bar and } 8 \mathrm{~mL} / \mathrm{min} \text { for } \\
\text { liquid temperature, pressure and } \\
\text { flow rate, respectively. }\end{array}$ & $99.9 \%(\mathrm{v} / \mathrm{v})$ & Karimi et al. [57]. \\
\hline $\begin{array}{l}\text { Regenerable } \\
\text { molecular sieve. }\end{array}$ & $\begin{array}{l}\text { Ethanol initial concentrations } \\
\text { between } 90-95 \mathrm{wt} \% \text {. and } 100 \mathrm{~min} \\
\text { of adsorption. }\end{array}$ & $93.62-97.71 \mathrm{wt} \%$ & Chen et al. [58]. \\
\hline $\begin{array}{l}\text { Distillation and } \\
\text { either adsorption } \\
\text { or pervaporation. }\end{array}$ & $\begin{array}{l}\text { Distillation: fermented liquid } \\
\text { containing ethanol } 6-12 \% \text { (v/v). } \\
\text { During distillation process (5.5 } \\
\text { h), cooler water was always } \\
\text { flowed into cooler to keep the } \\
\text { temperature at } 79{ }^{\circ} \mathrm{C} \text {. } \\
\text { Adsorption: zeolite } 3 \mathrm{~A} \text { and the } \\
\text { bioethanol resulted from } \\
\text { distillation unit with the } \\
\text { concentration of } 90-95 \% \text {. } \\
\text { Pervaporatión: the bioethanol was } \\
\text { pumped to the membrane module. } \\
\text { The pressure in permeate side was } \\
\text { adjusted at } 6 \text { mbar. After the } \\
\text { operating condition was achieved, } \\
\text { the process was operated for } 2 \\
\text { hours. }\end{array}$ & Higher than $99 \%$. & $\begin{array}{ll}\text { Kusmiyati } & \text { and } \\
\text { Susanto [59]. } & \end{array}$ \\
\hline Adsorption. & $\begin{array}{l}\text { Feeds containing } 80-95 \% \text { wt } \\
\text { ethanol at } 90-110{ }^{\circ} \mathrm{C} \text { and } 136-243 \\
\mathrm{kPa}\end{array}$ & $99 \%$ wt & Ranjbar et al. [60] \\
\hline $\begin{array}{l}\text { Pressure Swing } \\
\text { Adsorption } \\
\text { (PSA). }\end{array}$ & $87.0 \%$ wt ethanol. & $99.5 \mathrm{wt} \%$ & Jeong et al. [61]. \\
\hline
\end{tabular}




\section{Process integration in bioethanol production}

Sharifah Soplah Syed Abdullah, Yoshihito Shirai, Ahmad Amiruddin Mohd Ali, Mahfuzah Mustapha and Mohd Ali Hassan, evaluated the production of bioethanol from petiole sugars of the oil palm within an integrated palm biomass biorefinery, obtaining that for 33.9 million liters/year of bioethanol production, the specific cost of production Of bioethanol is estimated at $\$ 0.52 / 1$, and bioethanol produced from sugarcane ranges between $\$ 0.31-0.34 / 1$ and that from other lignocellulosic wastes between \$ 0.49-0, 60/1 [1]. Jegannathan Kenthorai Raman and Edgard Gnansounou evaluated ethanol production using Aspen Plus and Aspen Process Economic Analyzer through a techno-economic analysis based on four lignocellulosic residues, sugarcane bagasse, coffee cut-stems, rice husks and empty fruit bunches in Colombia. They found that the empty fruit bunches show the highest ethanol yield $(313.83 \mathrm{~L} / \mathrm{t})$ and the rice husk $(250.56 \mathrm{~L} / \mathrm{t})$. On the other hand, the economic analysis showed that the cost of production of ethanol with empty fruit bunches was the lowest with (0.49 US \$/L) and without (0.58 US \$/L) of cogeneration scheme [2]. Table 3 shows the advances in mass and energy integration processes.

Table 3.Advances in mass and energy integration processes for bioethanol production

\begin{tabular}{|c|c|c|c|}
\hline Approach & Conditions & Important conclusions & References \\
\hline $\begin{array}{l}\text { The bioethanol production } \\
\text { from oil palm frond (OPF) } \\
\text { petiole sugars within an } \\
\text { integrated palm biomass } \\
\text { biorefinery was carried out }\end{array}$ & $\begin{array}{l}\text { The integrated biorefinery will } \\
\text { be located at one of the } 4 \text { mills. }\end{array}$ & $\begin{array}{l}\text { The specific production } \\
\text { cost of bioethanol is } \\
\text { estimated at } \$ 0.52 / 1 \\
\text { bioethanol. }\end{array}$ & {$[61]$} \\
\hline $\begin{array}{l}\text { The ethanol production was } \\
\text { evaluated using Aspen Plus } \\
\text { and Aspen Process } \\
\text { Economic Analyzer } \\
\text { carrying out the simulation } \\
\text { and the economic } \\
\text { evaluation, respectively. }\end{array}$ & $\begin{array}{l}\text { The ethanol production cost } \\
\text { was assessed for the standalone } \\
\text { ethanol plant and the ethanol } \\
\text { plant coupled with a } \\
\text { cogeneration system }\end{array}$ & $\begin{array}{l}\text { Ethanol production cost } \\
\text { using Empty fruit bunches } \\
\text { was the lowest with }(0.49 \\
\text { US\$/L) and without }(0.58 \\
\text { US\$/L) rogeneration } \\
\text { scheme. }\end{array}$ & {$[62]$} \\
\hline $\begin{array}{l}\text { A hierarchical four-level } \\
\text { approach to determine } \\
\text { economic potential that } \\
\text { includes input/output } \\
\text { structure, process flow } \\
\text { structure, heat integration } \\
\text { (HI), and economic } \\
\text { feasibility. }\end{array}$ & $\begin{array}{l}\text {-A bioethanol plant with a } \\
\text { production of } 30.2 \mathrm{t} / \mathrm{d} \text {. }\end{array}$ & $\begin{array}{l}\text { The heating energy was } \\
\text { reduced considerably by } \\
\text { heat integration. The value } \\
\text { of bioethanol was estimated } \\
\text { at } \$ 0.8-\$ 1.3 / \mathrm{kg} \text {. }\end{array}$ & [63] \\
\hline $\begin{array}{l}\text { A hierarchical four level } \\
\text { economic potential } \\
\text { approach for: bioethanol } \\
\text { and jet fuel by } \\
\text { bioconversion, combined } \\
\text { heat and power via } \\
\text { gasification, the heat } \\
\text { integration (HI), and the } \\
\text { economic feasibility. }\end{array}$ & $\begin{array}{l}\text { Three energy conversions from } \\
400 \text { t/d wet to empty fruit } \\
\text { bunches. }\end{array}$ & $\begin{array}{l}\text { The biooil hydrocarbon } \\
\text { plant is most economical } \\
\text { due to the highest economic } \\
\text { potential. }\end{array}$ & [64] \\
\hline $\begin{array}{l}\text { Integration of flows, } \\
\text { materials and energy of oil } \\
\text { extracted from fresh fruit } \\
\text { bunches as raw material for } \\
\text { the production of biodiesel } \\
\text { and bioethanol }\end{array}$ & Ethanol production in situ & $\begin{array}{l}\text { A reduction in unit energy } \\
\text { costs down to } 3.4 \% \text {, } \\
\text { whereas the material and } \\
\text { energy integration leaded to } \\
39.8 \% \text { decrease of those } \\
\text { costs. }\end{array}$ & [65] \\
\hline $\begin{array}{l}\text { To used computational } \\
\text { fluid dynamics and an } \\
\text { integrated system. }\end{array}$ & $\begin{array}{l}\text {-Mass fractions of } 0 \%, 10 \%, \\
25 \% \text { and } 50 \% \text { to } \\
\text { hydrothermally treatment. }\end{array}$ & $\begin{array}{l}\text { Low energy consumption } \\
\text { during coal drying and } \\
\text { hydrothermally treatment of }\end{array}$ & [66] \\
\hline
\end{tabular}




\begin{tabular}{|c|c|c|c|}
\hline & $\begin{array}{l}\text { - To evaluated coal drying, } \\
\text { hydrothermally treatment of } \\
\text { Empty fruit bunches, cofiring, } \\
\text { and power generation }\end{array}$ & $\begin{array}{l}\text { Empty fruit bunches can be } \\
\text { achieved. }\end{array}$ & \\
\hline $\begin{array}{l}\text { An improved process } \\
\text { integration technology that } \\
\text { includes: drying, } \\
\text { gasification and combined } \\
\text { cycle processes for the use } \\
\text { of Empty fruit bunches }\end{array}$ & $\begin{array}{l}\text { Used a fluidized bed dryer with } \\
\text { superheated steam as the main } \\
\text { evaporator. }\end{array}$ & $\begin{array}{l}\text { The integrated drying can } \\
\text { further reduce the energy } \\
\text { consumption in drying up } \\
\text { to about } 30 \% \text { compared to a } \\
\text { standalone drying process } \\
\text { employing exergy recovery. }\end{array}$ & [67] \\
\hline $\begin{array}{l}\text { To investigate the } \\
\text { economic convenience of } \\
\text { supercritical } \\
\text { gasification technology } \\
\text { applied to a potential } \\
\text { industrial case study with } \\
\text { Aspen plus } 7.2\end{array}$ & $\begin{array}{l}\text { The feedstock is } 35 \mathrm{Mg} / \mathrm{h} \text { of } \\
\text { empty fruit bunches. }\end{array}$ & $\begin{array}{l}\text { The results show a great } \\
\text { benefit of the purposed } \\
\text { solutions and their } \\
\text { environmental } \\
\text { sustainability. }\end{array}$ & {$[68]$} \\
\hline $\begin{array}{l}\text { Innovative design of } \\
\text { production of crude palm } \\
\text { oil and palm oil based on } \\
\text { process } \\
\text { technology }\end{array}$ & $\begin{array}{l}\text { Considered a conventional } \\
\text { boiler based cogeneration } \\
\text { system and an internal } \\
\text { combustion engine based } \\
\text { cogeneration system. }\end{array}$ & $\begin{array}{l}\text { The significant energy } \\
\text { surplus in both processes }\end{array}$ & [69] \\
\hline $\begin{array}{l}\text { The unit operations are } \\
\text { simulated using commercial } \\
\text { software, Aspen Plus V7.1, } \\
\text { which is a standard process } \\
\text { simulation tool and has } \\
\text { been widely adopted to } \\
\text { simulate biomass CHP } \\
\text { (Combined Heat and } \\
\text { Power) systems. }\end{array}$ & $\begin{array}{l}\text { In the first stage of pretreatment } \\
\text { process, the sago biomass is fed } \\
\text { to a pretreatment reactor and } \\
\text { mixed with diluted sulphuric } \\
\text { acid ( } 18 \mathrm{mg} \text { acid/dry g of } \\
\text { biomass) that catalysis the } \\
\text { hydrolysis reaction at a } \\
\text { temperature of } 158{ }^{\circ} \mathrm{C} \text {. High } \\
\text { pressure ( } 13 \text { bar) steam is used } \\
\text { in this stage to maintain the } \\
\text { temperature. }\end{array}$ & $\begin{array}{l}\text { By using combined } \\
\text { biomass in the integrated } \\
\text { SBB with onsite enzyme } \\
\text { production and making use } \\
\text { of existing man power its } \\
\text { economic performance can } \\
\text { be improved ( } 6.6 \text { years of } \\
\text { payback period). }\end{array}$ & [70] \\
\hline $\begin{array}{l}\text { The diluted acid was } \\
\text { selected for pretreatment } \\
\text { stage of sugarcane bagasse } \\
\text { and three bioethanol } \\
\text { production topologies were } \\
\text { simulated through ASPEN } \\
\text { PLUS }\end{array}$ & $\begin{array}{l}\text { Saccharification/fermentation: } \\
101.325 \mathrm{kPa}, 321 \mathrm{~K} \text {, } \\
\text { Cellulase enzyme } 20 \mathrm{FPU} / \mathrm{g} \\
\text { cellulose/101.325 KPa, } 303 \mathrm{~K} \\
\text { S. Cervisiase. } \\
\text { Purification: molecular sieves }\end{array}$ & $\begin{array}{l}\text { Designs of heat exchange } \\
\text { network for thermal } \\
\text { integration of the process } \\
\text { were used to reduce the } \\
\text { consumption of natural } \\
\text { resources, reduce operating } \\
\text { cost, and enhance } \\
\text { environmental } \\
\text { performance. }\end{array}$ & {$[71]$} \\
\hline $\begin{array}{l}\text { The biorefinery used in this } \\
\text { work is the process for first } \\
\text { and } \\
\text { second generation }(1 \mathrm{G} / 2 \mathrm{G}) \\
\text { ethanol and bioelectricity } \\
\text { production }\end{array}$ & \begin{tabular}{ll}
\multicolumn{2}{l}{ Hydrothermal pretreatment } \\
consists in contact of \\
lignocellulosic
\end{tabular} & $\begin{array}{l}\text { Presented results indicate } \\
\text { that energy integration } \\
\text { provided considerable } \\
\text { reduction in energy } \\
\text { consumption and } \\
\text { consequently in operating } \\
\text { costs of the plant for all } \\
\text { evaluated scenarios. }\end{array}$ & {$[72]$} \\
\hline $\begin{array}{l}\text { The application of a linear } \\
\text { programming (LP) } \\
\text { cooperative game model to } \\
\text { allocate benefits that accrue } \\
\text { from interplant integration }\end{array}$ & $\begin{array}{l}\text { A case study of the literature } \\
\text { and the results are compared to } \\
\text { those determined through } \\
\text { alternative techniques of } \\
\text { cooperative play with two }\end{array}$ & $\begin{array}{l}\text { The model allowsto } \\
\text { determine the optimum } \\
\text { configuration and the } \\
\text { distribution of benefits in a } \\
\text { single step. }\end{array}$ & [73] \\
\hline
\end{tabular}




\begin{tabular}{|c|c|c|c|}
\hline in an Eco industrial park & $\begin{array}{l}\text { industrial case studies on the } \\
\text { integration of interplants in the } \\
\text { palm based biomass processing } \\
\text { complex and sago-based } \\
\text { biorefinery (SBB). }\end{array}$ & & \\
\hline $\begin{array}{l}\text { Extractive dividing-wall } \\
\text { column and pressure swing } \\
\text { adsorption processes for } \\
\text { bioethanol } \\
\text { recovery/purification are } \\
\text { simulated in Aspen HYSYS } \\
\text { v8.2 }\end{array}$ & $\begin{array}{l}\text {-The realistic feed stream of } 10 \\
\text { wt } \% \text { ethanol, } 89.9 \text { wt } \% \text { water } \\
\text { and } 0.1 \mathrm{wt} \% \text { carbon dioxide. } \\
\text {-Pressure swing adsorption } \\
\text { process involves distillation for } \\
\text { removing most of water, before } \\
\text { dehydration by pressure swing } \\
\text { adsorption }\end{array}$ & $\begin{array}{l}\text { Pressure swing adsorption } \\
\text { process has } 33 \% \text { lower cost } \\
\text { of manufacture per unit } \\
\text { product than extractive } \\
\text { dividing-wall column } \\
\text { process. }\end{array}$ & [74] \\
\hline $\begin{array}{l}\text { To simulations of an } \\
\text { autonomous distillery were } \\
\text { carried out, along with } \\
\text { utilities demand } \\
\text { optimization using Pinch } \\
\text { Analysis concepts with the } \\
\text { use of the commercial } \\
\text { software UniSim Design. }\end{array}$ & $\begin{array}{l}\text {-Pressure } 80 \text { bar } \\
\text {-Temperature } 510^{\circ} \mathrm{C} \\
\text {-The next system: a traditional } \\
\text { Rankine Cycle and a Biomass } \\
\text { Integrated Gasification } \\
\text { Combined Cycle }\end{array}$ & $\begin{array}{l}\text { The use of thermal } \\
\text { integration techniques } \\
\text { allows a reduction of the } \\
\text { steam process and } \\
\text { consequently produces } \\
\text { more bagasse surplus or } \\
\text { increases the excess } \\
\text { electricity }\end{array}$ & [75] \\
\hline $\begin{array}{l}\text { The second-generation } \\
\text { ethanol modelled through a } \\
\text { process simulation } \\
\text { developed in the software } \\
\text { Aspen Plus environment } \\
\text { and performed an analysis } \\
\text { of the conversion } \\
\text { technologies in terms of } \\
\text { yield, efficiency, feedstock } \\
\text { and level of process } \\
\text { integration. }\end{array}$ & $\begin{array}{l}\text { Efficient hexose and pentose } \\
\text { fermentation } \\
\text {-Enzyme strain development } \\
\text { and solid co-product } \\
\text { valorization for process heat } \\
\text { and power generation. } \\
\text { A production capacity of about } \\
40,000 \text { tethanol/y for the plant. } \\
\text { and total energy input of } 108 \\
\text { MW, } 36 \mathrm{MW} \text {. }\end{array}$ & $\begin{array}{l}\text { The net process energy } \\
\text { efficiency is calculated in } \\
\text { the range from } 35 \% \text { to } 37 \%\end{array}$ & {$[76]$} \\
\hline $\begin{array}{l}\text { A computational tool } \\
\text { developed within an } \\
\text { equation-oriented process } \\
\text { simulator that couples the } \\
\text { simulation of first and } \\
\text { second-generation } \\
\text { bioethanol production with } \\
\text { a global optimization } \\
\text { algorithm }\end{array}$ & $\begin{array}{l}\text { Sugarcane bagasse is already } \\
\text { used mainly for supplying } \\
\text { electric and thermal energy to } \\
\text { the process itself }\end{array}$ & $\begin{array}{l}\text { The production of second } \\
\text { generation } \\
\text { increases thermal demands } \\
\text { in at least } 25 \% \text {. } \\
\text { The electric power surplus } \\
\text { is diminished in at least } \\
31 \%\end{array}$ & [77] \\
\hline $\begin{array}{l}\text { Aspen Plus was used to } \\
\text { investigate the energy } \\
\text { requirement of a } \\
\text { configuration of double- } \\
\text { effect forward-integrated } \\
\text { columns and response } \\
\text { surface methodology was } \\
\text { applied to optimize the } \\
\text { process and analyze some } \\
\text { operating parameters }\end{array}$ & $\begin{array}{l}\text { An extra stripping section to } \\
\text { reduce the risk of fouling in the } \\
\text { process. Multicomponent } \\
\text { mixture in the process. }\end{array}$ & $\begin{array}{l}\text { Utility costs were } 30.9 \% \text { in } \\
\text { the former design and } \\
32.4 \% \text { in the latter }\end{array}$ & [78] \\
\hline $\begin{array}{l}\text { A bioethanol integration } \\
\text { based on mass pinch } \\
\text { analysis for the analysis and } \\
\text { design of product exchange } \\
\text { networks formed in }\end{array}$ & $\begin{array}{l}\text { The combination of analytical- } \\
\text { graphical and cost-benefit } \\
\text { analysis for facilitate the whole } \\
\text { bioethanol based biorefinery. } \\
\text { A biorefinery producing }\end{array}$ & $\begin{array}{l}\text { Integrated } r \text { bioethanol } \\
\text { exchange networks are } \\
\text { effective in preventing } \\
\text { significant loss of revenue } \\
\text { in the processes involved }\end{array}$ & [79] \\
\hline
\end{tabular}




\begin{tabular}{|c|c|c|c|}
\hline $\begin{array}{l}\text { biorefinery pathways } \\
\text { featuring a set of processing } \\
\text { units producing or utilizing } \\
\text { bioethanol. }\end{array}$ & $\begin{array}{l}\text { bioethanol from wheat with } \\
\text { arabinoxylan (AX) co- } \\
\text { production using bioethanol for } \\
\text { AX precipitation. }\end{array}$ & & \\
\hline $\begin{array}{l}\text { An analysis for the } \\
\text { separation process in } \\
\text { bioethanol production } \\
\text { incorporating mass and } \\
\text { energy integration where, } \\
\text { energy integration used } \\
\text { SYNHEAT optimization } \\
\text { model and for the mass } \\
\text { integration process a direct } \\
\text { recycle strategy }\end{array}$ & $\begin{array}{l}\text { Optimized azeotropic } \\
\text { separation processes in } \\
\text { conjunction with process } \\
\text { integration. Utility cost savings, } \\
\text { energy requirement savings, } \\
\text { and increased area of the } \\
\text { sequences with mass and } \\
\text { energy integration for a best } \\
\text { bioethanol separation process }\end{array}$ & $\begin{array}{l}\text {-The conventional sequence } \\
\text { had savings of } 16.75 \% \text { in } \\
\text { utility costs and } 96 \% \text { of } \\
\text { mass integration of solvent, } \\
\text { and to the optional } \\
\text { sequence OSS-EI-III had } \\
\text { savings of } 5.48 \% \text { in utility } \\
\text { costs due to energy } \\
\text { integration and mass } \\
\text { integration of } 97 \% \text { solvent }\end{array}$ & {$[80]$} \\
\hline $\begin{array}{l}\text { A hybrid thermal } \\
\text { integration scheme is } \\
\text { proposed for a pressure- } \\
\text { swing distillation column } \\
\text { by combining an internally } \\
\text { heat integrated distillation } \\
\text { column with vapor } \\
\text { recompression column }\end{array}$ & $\begin{array}{l}\text { The pressure-swing distillation } \\
\text { is a conventional standalone. } \\
\text { The two performance indexes } \\
\text { for the estimated, namely } \\
\text { energy consumption and total } \\
\text { annual cost. }\end{array}$ & $\begin{array}{l}\text { The hybrid integrated } \\
\text { thermal internal distillation } \\
\text { column configuration } \\
\text { showing a promising } \\
\text { performance from the } \\
\text { standpoint of energy } \\
(82.88 \%), \text { utility cost } \\
(64.05 \%) \text { and savings } \\
(22.16 \%)\end{array}$ & [81] \\
\hline $\begin{array}{l}\text { To evaluate the reduction of } \\
\text { process steam demand and } \\
\text { water usage obtained } \\
\text { through heat integration } \\
\text { and an exergy analysis to } \\
\text { quantify the reduction in } \\
\text { irreversibility generation } \\
\text { owing to heat integration } \\
\text { procedure using the } \\
\text { software Aspen Plus and } \\
\text { pinch method. }\end{array}$ & $\begin{array}{l}\text { Two cases of study: Case I: all } \\
\text { sugarcane juice is destined to } \\
\text { produce ethanol. } \\
\text { Case II: } 50 \% \text { sugar production } \\
\text { and } 50 \% \text { ethanol production. }\end{array}$ & $\begin{array}{l}\text { The heat integration was } \\
\text { reduced in terms of the } \\
\text { steam consumption in } 35 \% \text {. } \\
\text { The reduction in water } \\
\text { consumption was } 24 \% \text { and } \\
13 \% \text { compared to } \\
\text { conventional cases without } \\
\text { heat integration. }\end{array}$ & [82] \\
\hline
\end{tabular}

\section{Acknowledgments}

The authors recognized to the University of Cartagena for providing the bibliographic databases for the development of this review article. 


\section{References}

1. M. S. Siti Aisyah, Y. Uemura, and S. Yusup, "The Effect of Alkaline Addition in Hydrothermal Pretreatment of Empty Fruit Bunches on Enzymatic Hydrolysis Efficiencies," Procedia Chem., vol. 9, pp. 151-157, 2014.

2. S. Kim and C. H. Kim, "Bioethanol production using the sequential acid/alkali-pretreated empty palm fruit bunch fiber," Renew. Energy, vol. 54, pp. 150-155, 2013.

3. M. M. Ishola, Isroi, and M. J. Taherzadeh, "Effect of fungal and phosphoric acid pretreatment on ethanol production from oil palm empty fruit bunches (OPEFB)," Bioresour. Technol., vol. 165, no. C, pp. 9-12, 2014.

4. Y. H. Jung, I. J. Kim, J. I. Han, I. G. Choi, and K. H. Kim, “Aqueous ammonia pretreatment of oil palm empty fruit bunches for ethanol production," Bioresour. Technol., vol. 102, no. 20, pp. 98069809, 2011.

5. Y. H. Jung, I. J. Kim, H. K. Kim, and K. H. Kim, "Dilute acid pretreatment of lignocellulose for whole slurry ethanol fermentation," Bioresour. Technol., vol. 132, pp. 109-114, 2013.

6. D. Carrillo-Nieves, L. Zumalacárregui-de-Cárdenas, R. Franco-Rico, and I. Sarvari-Horvath, "Kinetic of biogas production from oil palm empty fruit bunches," Dyna, vol. 81, no. 187, pp. 96$101,2014$.

7. M. Misson, R. Haron, M. F. A. Kamaroddin, and N. A. S. Amin, "Pretreatment of empty palm fruit bunch for production of chemicals via catalytic pyrolysis," Bioresour. Technol., vol. 100, no. 11, pp. 2867-2873, 2009.

8. A. Kristiani, N. Effendi, Y. Aristiawan, F. Aulia, and Y. Sudiyani, "Effect of Combining Chemical and Irradiation Pretreatment Process to Characteristic of Oil Palm's Empty Fruit Bunches as Raw Material for Second Generation Bioethanol," Energy Procedia, vol. 68, pp. 195-204, 2015.

9. S. Chiesa and E. Gnansounou, "Use of empty fruit bunches from the oil palm for bioethanol production: A thorough comparison between dilute acid and dilute alkali pretreatment," Bioresour. Technol., vol. 159, pp. 355-364, 2014.

10. J. D. C. Medina, A. Woiciechowski, A. Z. Filho, P. S. Nigam, L. P. Ramos, and C. R. Soccol, "Steam explosion pretreatment of oil palm empty fruit bunches (EFB) using autocatalytic hydrolysis: A biorefinery approach," Bioresour. Technol., vol. 199, pp. 173-180, 2016.

11. J. D. Coral Medina et al., "Biological activities and thermal behavior of lignin from oil palm empty fruit bunches as potential source of chemicals of added value," Ind. Crops Prod., vol. 94, pp. 630637, 2016.

12. A. A. Kamoldeen, C. K. Lee, W. N. Wan Abdullah, and C. P. Leh, "Enhanced ethanol production from mild alkali-treated oil-palm empty fruit bunches via co-fermentation of glucose and xylose," Renew. Energy, vol. 107, pp. 113-123, 2017.

13. M. J. Lau, M. W. Lau, C. Gunawan, and B. E. Dale, "Ammonia fiber expansion (AFEX) pretreatment, enzymatic hydrolysis, and fermentation on empty palm fruit bunch fiber (EPFBF) for cellulosic ethanol production," Appl. Biochem. Biotechnol., vol. 162, no. 7, pp. 1847-1857, 2010.

14. M. Han, Y. Kim, S. W. Kim, and G. W. Choi, "High efficiency bioethanol production from OPEFB using pilot pretreatment reactor," J. Chem. Technol. Biotechnol., vol. 86, no. 12, pp. 1527-1534, 2011.

15. R. J. Bouza, Z. Gu, and J. H. Evans, "Screening conditions for acid pretreatment and enzymatic hydrolysis of empty fruit bunches," Ind. Crops Prod., vol. 84, pp. 67-71, 2016.

16. F. Fahma, S. Iwamoto, N. Hori, T. Iwata, and A. Takemura, "Isolation, preparation, and characterization of nanofibers from oil palm empty-fruit-bunch (OPEFB)," Cellulose, vol. 17, no. 5, pp. 977-985, 2010.

17. Y. Piñeros-Castro and M. Velásquez-Lozano, "Biodegradation kinetics of oil palm empty fruit bunches by white rot fungi," Int. Biodeterior. Biodegrad., vol. 91, pp. 24-28, 2014.

18. W. N. N. Wan Omar and N. A. S. Amin, "Multi response optimization of oil palm frond pretreatment by ozonolysis," Ind. Crops Prod., vol. 85, pp. 389-402, 2016.

19. L. Tan et al., "Pretreatment of empty fruit bunch from oil palm for fuel ethanol production and proposed biorefinery process," Bioresour. Technol., vol. 135, pp. 275-282, 2013.

20. L. Tan et al., "Bisulfite pretreatment changes the structure and properties of oil palm empty fruit bunch to improve enzymatic hydrolysis and bioethanol production," Biotechnol. J., vol. 10, no. 6, pp. 915-925, 2015. 
21. L. Tan et al., "Fractionation of oil palm empty fruit bunch by bisulfite pretreatment for the production of bioethanol and high value products," Bioresour. Technol., vol. 200, pp. 572-578, 2016.

22. S. X. Chin, C. H. Chia, S. Zakaria, Z. Fang, and S. Ahmad, "Ball milling pretreatment and diluted acid hydrolysis of oil palm empty fruit bunch (EFB) fibres for the production of levulinic acid," $J$. Taiwan Inst. Chem. Eng., vol. 52, pp. 85-92, 2015.

23. S. O-Thong, K. Boe, and I. Angelidaki, "Thermophilic anaerobic co-digestion of oil palm empty fruit bunches with palm oil mill effluent for efficient biogas production," Appl. Energy, vol. 93, pp. 648-654, 2012.

24. A. S. Baharuddin et al., "Selective component degradation of oil palm empty fruit bunches (OPEFB) using high-pressure steam," Biomass and Bioenergy, vol. 55, pp. 268-275, 2013.

25. Nurfahmi, H. C. Ong, B. M. Jan, C. W. Tong, H. Fauzi, and W. H. Chen, "Effects of organosolv pretreatment and acid hydrolysis on palm empty fruit bunch (PEFB) as bioethanol feedstock," Biomass and Bioenergy, vol. 95, pp. 78-83, 2016.

26. D. Piarpuzán, J. A. Quintero, and C. A. Cardona, "Empty fruit bunches from oil palm as a potential raw material for fuel ethanol production," Biomass and Bioenergy, vol. 35, no. 3, pp. 1130-1137, 2011.

27. F. B. Ahmad, Z. Zhang, W. O. S. Doherty, V. S. J. Te'o, and I. M. O'Hara, "Improved microbial oil production from oil palm empty fruit bunch by Mucor plumbeus," Fuel, vol. 194, pp. 180-187, 2017.

28. R. Yunus, S. F. Salleh, N. Abdullah, and D. R. A. Biak, "Effect of ultrasonic pre-treatment on low temperature acid hydrolysis of oil palm empty fruit bunch," Bioresour. Technol., vol. 101, no. 24, pp. 9792-9796, 2010.

29. T. Y. Ying, L. K. Teong, W. N. W. Abdullah, and L. C. Peng, "The Effect of Various Pretreatment Methods on Oil Palm Empty Fruit Bunch (EFB) and Kenaf Core Fibers for Sugar Production," Procedia Environ. Sci., vol. 20, pp. 328-335, 2014.

30. S. Kim, J. M. Park, J. W. Seo, and C. H. Kim, "Sequential acid-/alkali-pretreatment of empty palm fruit bunch fiber," Bioresour. Technol., vol. 109, pp. 229-233, 2012.

31. H. Jeon et al., "Production of anhydrous ethanol using oil palm empty fruit bunch in a pilot plant," Biomass and Bioenergy, vol. 67, pp. 99-107, 2014.

32. T. C. Coelho, O. Souza, N. Sellin, S. H. W. Medeiros, and C. Marangoni, "Analysis of the reflux ratio on the batch distillation of bioethanol obtained from lignocellulosic residue," Procedia Eng., vol. 42, no. August, pp. 131-139, 2012.

33. M. Tomaszewska and L. Białończyk, "Ethanol production from whey in a bioreactor coupled with direct contact membrane distillation," Catal. Today, vol. 268, pp. 156-163, 2016.

34. M. Tomaszewska and L. Bia, "Production of ethanol from lactose in a bioreactor integrated with membrane distillation," pp. 1-6, 2013.

35. M. Barancewicz and M. Gryta, "Ethanol production in a bioreactor with an integrated membrane distillation module," Chem. Pap., vol. 66, no. May 2011, pp. 85-91, 2012.

36. G. Lewandowicz, W. Białas, B. Marczewski, and D. Szymanowska, "Application of membrane distillation for ethanol recovery during fuel ethanol production," J. Memb. Sci., 2011.

37. D. Woldemariam, A. Kullab, E. U. Khan, and A. Martin, "Recovery of ethanol from scrubber-water by district heat-driven membrane distillation: Industrial-scale technoeconomic study," Renew. Energy, pp. 1-11, 2017.

38. M. M. A. Shirazi, A. Kargari, and M. Tabatabaei, "Sweeping Gas Membrane Distillation (SGMD) as an Alternative for Integration of Bioethanol Processing: Study on a Commercial Membrane and Operating Parameters," Chem. Eng. Commun., 2015.

39. Q. Zhang et al., "Ethanol production by modified polyvinyl alcohol-immobilized Zymomonas mobilis and in situ membrane distillation under very high gravity condition," Appl. Energy, vol. 202, pp. 1-5, 2017.

40. L. Zhang, Y. Wang, L. H. Cheng, X. Xu, and H. Chen, "Concentration of lignocellulosic hydrolyzates by solar membrane distillation," Bioresour. Technol., vol. 123, pp. 382-385, 2012.

41. U. M. García-Ventura, F. O. Barroso-Muñoz, S. Hernández, and A. J. Castro-Montoya, "Experimental study of the production of high purity ethanol using a semi-continuous extractive batch dividing wall distillation column," Chem. Eng. Process. Process Intensif., vol. 108, pp. 74-77, 2016. 
42. S. Navarrete-Contreras, M. Sánchez-Ibarra, F. O. Barroso-Muñoz, S. Hernández, and A. J. CastroMontoya, "Use of glycerol as entrainer in the dehydration of bioethanol using extractive batch distillation: Simulation and experimental studies," Chem. Eng. Process. Process Intensif., vol. 77, pp. 38-41, 2014.

43. J. ángel Pacheco-Basulto et al., "Purification of bioethanol using extractive batch distillation: Simulation and experimental studies," Chem. Eng. Process. Process Intensif., vol. 61, pp. 30-35, 2012.

44. J. Niemistö, A. Pasanen, K. Hirvelä, L. Myllykoski, E. Muurinen, and R. L. Keiski, "Pilot study of bioethanol dehydration with polyvinyl alcohol membranes," J. Memb. Sci., vol. 447, pp. 119-127, 2013.

45. C. Fu et al., "Ethanol fermentation integrated with PDMS composite membrane: An effective process," Bioresour. Technol., vol. 200, pp. 648-657, 2016.

46. H. J. Lee, E. J. Cho, Y. G. Kim, I. S. Choi, and H. J. Bae, "Pervaporative separation of bioethanol using a polydimethylsiloxane/polyetherimide composite hollow-fiber membrane," Bioresour. Technol., vol. 109, pp. 110-115, 2012.

47. S. Yi and Y. Wan, "Separation performance of novel vinyltriethoxysilane (VTES)-g-silicalite1/PDMS/PAN thin-film composite membrane in the recovery of bioethanol from fermentation broths by pervaporation," J. Memb. Sci., vol. 524, no. July 2016, pp. 132-140, 2017.

48. S. Fan, Z. Xiao, and M. Li, "Energy efficient of ethanol recovery in pervaporation membrane bioreactor with mechanical vapor compression eliminating the cold traps," Bioresour. Technol., vol. 211, pp. 24-30, 2016.

49. R. H. Bello, O. Souza, N. Sellin, S. H. W. Medeiros, and C. Marangoni, "Effect of operating variables on the pervaporation of ethanol produced by lignocellulosic residue," Procedia Eng., vol. 42, no. August, pp. 512-520, 2012.

50. M. Moussa, V. Athès, Y. Imbert, I. Souchon, O. Vitrac, and M. L. Lameloise, "Pervaporative dehydration of bioethanol using silica and PVA membranes: Analysis of permeation performances and effect of volatile organic impurities," Procedia Eng., vol. 44, pp. 1173-1176, 2012.

51. M. A. Pratiwi, R. W. Sudrajat, S. Sutanti, and H. Susanto, "Preparation of Chitosan-Alginate/PES Pervaporation Membranes for Bioethanol Dehydration," Adv. Mater. Res., vol. 1123, pp. 182-186, 2015.

52. Y. H. Huang et al., "A study on the characteristics and pervaporation performance of polyamide thin-film composite membranes with modified polyacrylonitrile as substrate for bioethanol dehydration," Polym. Int., vol. 63, no. 8, pp. 1478-1486, 2014.

53. S. K. Wahono, Hernawan, A. Kristiani, S. Tursiloadi, and H. Abimanyu, "Characterization and utilization of gunungkidul natural zeolite for bioethanol dehydration," Energy Procedia, vol. 47, pp. 263-267, 2014.

54. H. Zhou, D. Korelskiy, T. Leppäjärvi, M. Grahn, J. Tanskanen, and J. Hedlund, "Ultrathin zeolite X membranes for pervaporation dehydration of ethanol," J. Memb. Sci., vol. 399-400, pp. 106-111, 2012.

55. A. H. Abdullah, N. N. Zulkefli, A. S. Abd Aziz, and R. Mat, "Synthesis of zeolite Na-A from local kaolin for bioethanol purification," Indian J. Sci. Technol., vol. 9, no. 9, 2016.

56. S. Karimi, B. Ghobadian, M. R. Omidkhah, J. Towfighi, and M. Tavakkoli Yaraki, "Experimental investigation of bioethanol liquid phase dehydration using natural clinoptilolite," J. Adv. Res., vol. 7, no. 3, pp. 435-444, 2016.

57. W. C. Chen et al., "Optimizing the efficiency of anhydrous ethanol purification via regenerable molecular sieve," Appl. Energy, vol. 135, pp. 483-489, 2014.

58. Kusmiyati and H. Susanto, "Fuel Grade Bioethanol Production from Iles-iles (Amorphophaluscampanulatus) Tuber," Procedia Environ. Sci., vol. 23, no. Ictcred 2014, pp. 199206, 2015.

59. Z. Ranjbar, M. Tajallipour, C. H. Niu, and A. K. Dalai, "Water removal from ethanol vapor by adsorption on canola meal after protein extraction," Ind. Eng. Chem. Res., vol. 52, no. 40, pp. 14429-14440, 2013.

60. J. seong Jeong, H. Jeon, K. mo Ko, B. Chung, and G. W. Choi, "Production of anhydrous ethanol using various PSA (Pressure Swing Adsorption) processes in pilot plant," Renew. Energy, vol. 42, pp. 41-45, 2012.

61. S. S. S. Abdullah, Y. Shirai, A. A. M. Ali, M. Mustapha, and M. A. Hassan, "Case study: 
Preliminary assessment of integrated palm biomass biorefinery for bioethanol production utilizing non-food sugars from oil palm frond petiole," Energy Convers. Manag., vol. 108, pp. 233-242, 2016.

62. J. A. Quintero, J. Moncada, and C. A. Cardona, "Techno-economic analysis of bioethanol production from lignocellulosic residues in Colombia: A process simulation approach," Bioresour. Technol., vol. 139, pp. 300-307, 2013.

63. T. X. Do, Y. il Lim, S. Jang, and H. J. Chung, "Hierarchical economic potential approach for technoeconomic evaluation of bioethanol production from palm empty fruit bunches," Bioresour. Technol., vol. 189, pp. 224-235, 2015.

64. T. X. Do and Y. Il Lim, "Techno-economic comparison of three energy conversion pathways from empty fruit bunches," Renew. Energy, vol. 90, pp. 307-318, 2016.

65. L. F. Gutiérrez, Ó. J. Sánchez, and C. A. Cardona, "Process integration possibilities for biodiesel production from palm oil using ethanol obtained from lignocellulosic residues of oil palm industry," Bioresour. Technol., vol. 100, no. 3, pp. 1227-1237, 2009.

66. A. Darmawan, D. Budianto, M. Aziz, and K. Tokimatsu, "Retrofitting existing coal power plants through cofiring with hydrothermally treated empty fruit bunch and a novel integrated system," Appl. Energy, 2017.

67. M. Aziz, P. Prawisudha, B. Prabowo, and B. A. Budiman, "Integration of energy-efficient empty fruit bunch drying with gasification/combined cycle systems," Appl. Energy, vol. 139, pp. 188-195, 2015.

68. S. Langè and L. A. Pellegrini, "Economic analysis of a combined production ofhydrogen-energy from empty fruit bunches," Biomass and Bioenergy, vol. 59, no. 0, pp. 520-531, 2013.

69. M. Aziz, T. O. Da, and T. K. Ashiwagi, "Design and Analysis of Energy-Efficient Integrated Crude Palm Oil and Palm Kernel Oil Processes," pp. 143-150, 2015.

70. Y. K. Wan, J. Sadhukhan, and D. K. S. Ng, "Techno-economic evaluations for feasibility of sagobased biorefinery, Part 2: Integrated bioethanol production and energy systems," Chem. Eng. Res. Des., vol. 107, pp. 102-116, 2016.

71. K. Ojeda, E. Sánchez, M. El-Halwagi, and V. Kafarov, "Exergy analysis and process integration of bioethanol production from acid pre-treated biomass: Comparison of SHF, SSF and SSCF pathways," Chem. Eng. J., vol. 176-177, pp. 195-201, 2011.

72. C. M. Oliveira, A. J. G. Cruz, and C. B. B. Costa, "Improving second generation bioethanol production in sugarcane biorefineries through energy integration," Appl. Therm. Eng., vol. 109, pp. 819-827, 2016.

73. R. R. Tan, V. Andiappan, Y. K. Wan, R. T. L. Ng, and D. K. S. Ng, "An optimization-based cooperative game approach for systematic allocation of costs and benefits in interplant process integration," Chem. Eng. Res. Des., vol. 106, pp. 43-58, 2016.

74. Y. Y. Loy, X. L. Lee, and G. P. Rangaiah, "Bioethanol recovery and purification using extractive dividing-wall column and pressure swing adsorption: An economic comparison after heat integration and optimization," Sep. Purif. Technol., vol. 149, pp. 413-427, 2015.

75. M. O. S. Dias, M. Modesto, A. V. Ensinas, S. A. Nebra, R. M. Filho, and C. E. V Rossell, "Improving bioethanol production from sugarcane: Evaluation of distillation, thermal integration and cogeneration systems," Energy, vol. 36, no. 6, pp. 3691-3703, 2011.

76. G. F. Porzio, M. Prussi, D. Chiaramonti, and L. Pari, "Modelling lignocellulosic bioethanol from poplar: Estimation of the level of process integration, yield and potential for co-products," J. Clean. Prod., vol. 34, pp. 66-75, 2012.

77. F. F. Furlan, C. B. B. Costa, G. de C. Fonseca, R. de P. Soares, A. R. Secchi, A. J. G. da Cruz, and R. de C. Giordano, "Assessing the production of first and second generation bioethanol from sugarcane through the integration of global optimization and process detailed modeling," Comput. Chem. Eng., vol. 43, pp. 1-9, 2012.

78. L. C. B. A. Bessa, M. C. Ferreira, E. A. C. Batista, and A. J. A. Meirelles, "Performance and cost evaluation of a new double-effect integration of multicomponent bioethanol distillation," Energy, vol. 63, pp. 1-9, 2013.

79. E. Martinez-Hernandez, J. Sadhukhan, and G. M. Campbell, "Integration of bioethanol as an inprocess material in biorefineries using mass pinch analysis," Appl. Energy, vol. 104, pp. 517-526, 2013.

80. M. V??zquez-Ojeda, J. G. Segovia-Hern??ndez, and J. M. Ponce-Ortega, "Incorporation of mass and 
energy integration in the optimal bioethanol separation process," Chem. Eng. Technol., vol. 36, no. 11, pp. 1865-1873, 2013.

81. B. Kiran and A. K. Jana, "A hybrid heat integration scheme for bioethanol separation through pressure-swing distillation route,” Sep. Purif. Technol., vol. 142, pp. 307-315, 2015.

82. E. A. Pina, R. Palacios-Bereche, M. F. Chavez-Rodriguez, A. V. Ensinas, M. Modesto, and S. A. Nebra, "Reduction of process steam demand and water-usage through heat integration in sugar and ethanol production from sugarcane - Evaluation of different plant configurations," Energy, 2015. 
Extra page Not to be printed.

For your Research References Requirements, Always log on to =www.sphinxsai.com

International Journal of ChemTech Research

[www.sphinxsai.com]

International Journal of TechnoChem Research [www.technochemsai.com]

$* * * * *$ 